\title{
17. COMMISSION DU MOUVEMENT ET DE LA \\ FIGURE DE LA LUNE
}

\section{Report of Meetings, 28 and 31 August 1964}

President: K. Koziel.

SeCRETARY: Flora McBain Sadler.

The Draft Report was approved without modification.

The President informed the Commission that he had received a letter from the Executive Committee of the Union requesting that consideration should be given to the place in the structure of the Union of the future work of the Commission. The first meeting, on 28 August 1964, was devoted to this question and the President opened the discussion by presenting the conception of expanding the scope of activity of Commission 17 over all aspects of lunar research.

A lively discussion followed in which Kopal, Arthur, O'Keefe, Markowitz, McBain Sadler, Eckert, Dollfus and Weimer took part. As a result, it was decided unanimously to recommend to the Executive Committee that Commission 17 should have restored to it its original title of 'La Lune' and have its responsibilities expanded to cover all aspects of lunar research. The dynamical and physical aspects of lunar research have become so closely interwoven that it is now necessary to combine all lunar work in one commission.

At the scientific meeting, on $3^{1}$ August $1964, D . W$. G. Arthur gave an account of the selenodetic programme of the Lunar and Planetary Laboratory of the University of Arizona. It is based on some 2000 lunar photographs obtained with the Yerkes 40-inch refractor in the years 1958 , 1960 and 1961 . About 500 of these are considered to be of useful quality. In addition about roo star-trailed lunar photographs were obtained for the investigation of the physical librations. The measuring machine is a Mann $422-\mathrm{C}$ equipped with Coleman digitizers. The readings are to $\mathrm{I}$ micron but the machine errors may amount to \pm 3 microns. Experience with good quality Yerkes plates indicates a standard value of \pm 7 microns for the reproducibility of single measures of the co-ordinates.

For the determination of lunar absolute altitudes, about 30 of the best plates will be used applying a modification of Saunders's method. Later a pure photogrammetric method will be used, making no use of the libration theory. About 2000 points have been selected for measurement in this programme.

In the programme for the physical librations, all roo star-trailed plates will be used. The observational equations will state the differences of co-ordinates between Mösting $\mathrm{A}$ and points close to but not on the limb. About $3 \circ$ points have been selected for measurement, five of which are near Mösting A and the remainder close to the limb.

In both the photogrammetric programme for the absolute altitudes and the programme for the physical librations, the focal length of the telescope will play a critical role. This length must be determined with a higher degree of accuracy than is usually required. For this purpose a special star-trail camera is under construction. In it the plate is oscillated in declination through I-5 mm, with a period of I second. The focal length may then be determined from the mean wavelength of the sinusoidal star-trail.

R. L. Duncombe described how, as part of the programme for observational re-determination of the constants of the physical libration of the Moon being carried out by the Nautical Almanac 
Office, U.S. Naval Observatory, a test was made of the systematic effects on the reduction of introducing Watts's limb corrections as compared to Hayn's limb corrections.

To make this test, the Dorpat heliometer observations of I 884 and I 885 were chosen. These observations have been completely discussed and rigorously reduced by $\mathrm{K}$. Koziel as clearly described in his series of papers in Acta Astronomica, Series A, volume 4, 1948-49. The reduced observations, as presented there, were modified by the introduction of Watts's limb corrections and a new solution made. As a control on the process of reforming the observational equations and normal equations, a solution without limb corrections was also made.

The results of the solution without limb corrections is shown at the top of Table A. The symbols $\lambda, \beta$ and $h$ represent the selenographic longitude and latitude of Mösting $A$ and its radius vector, i.e. its distance from the centre of the Moon at the mean distance of the Moon from the Earth. $I$ is the angle between the Moon's equator and the ecliptic, $f=\alpha / \beta$ is the mechanical ellipticity of the Moon, and $R$ is the radius of the Moon. The insignificant differences from the same solution made by Koziel arise from assigning unit weight to all the observational equations, and to small differences in decimal accuracy of the computations.

Table A

\section{Solution without Limb Corrections}

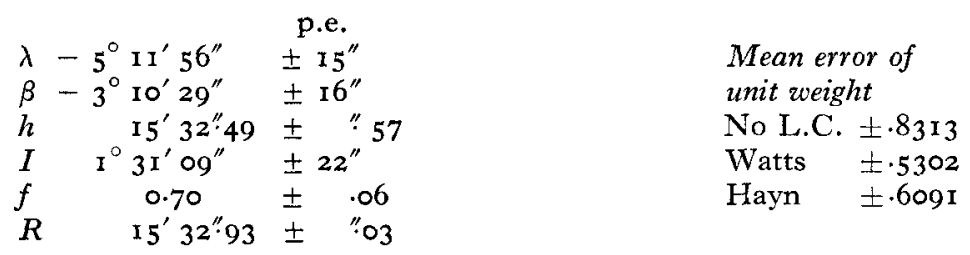

\section{Solution with Limb Corrections}

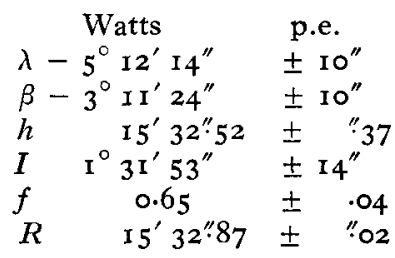

$$
\begin{gathered}
\text { Hayn } \\
-5^{\circ} \text { I } 1^{\prime} 50^{\prime \prime} \\
-3^{\circ} 10^{\prime} 27^{\prime \prime} \\
\text { I } 5^{\prime} 32^{\prime \prime} 88 \\
1^{\circ} 3 \mathbf{1}^{\prime} 36^{\prime \prime} \\
0.71 \\
\text { I } 5^{\prime} 32^{\prime \prime} 88
\end{gathered}
$$

The results of the solution using Watts's limb corrections is given in the lower left. For comparison, the results of the previous solution using Hayn's limb corrections is shown to the right.

The mean error of unit weight of the three solutions is shown in the upper right portion of Table A. While the error using Watts's limb corrections is less than when using Hayn's limb corrections, there appear to be residual errors in the observations themselves that are larger than the limb effects.

The systematic shift in latitude introduced by Watts in forming his definitive datum surface (Astronomical Papers, $\mathbf{1 7}, 95_{1}^{1}, 1963$ ) is reflected in the latitude of Mösting A resulting from the solution using his limb corrections. A slightly different value of the inclination of the Moon's equator to the ecliptic also is apparent.

For lack of sufficient observational weight, no discussion of the derived value of $f$ is given, nor has any attempt been made to solve for the coefficients of the free libration in longitude.

In the discussion which followed, Koziel remarked that he had expanded his work to include 
four other series and hoped to find that this reduced the probable errors significantly. Duncombe pointed out that his work was intended to find the effect on the probable errors of introducing Watts's corrections. They had hoped for a marked reduction in the values of the probable errors but the effect was not great. It is intended to re-measure the plates used by Watts. Some further discussion on the resolution of the photographs used by Watts took place, Kopal, Duncombe, O'Keefe and Arthur taking part.

$W . \mathcal{F}$. Eckert said he had already reported to Commission 7 on the results of a new solution of the 'main problem' of the lunar theory which has been made by H. J. Smith, Jr. and himself. But of particular interest to Commission 17 is the derived information concerning the distribution of mass in the Moon resulting from the new values of the motions of the perigee and node. The results are

$\begin{array}{lcc}\frac{1}{k} & \frac{3}{2} \frac{C}{M D^{2}} & \frac{C-B}{C-A} \\ 298 & 0.87 & 0.68 \\ 298.25 & 0.90 & 0.69\end{array}$

where $k$ is the oblateness of the Earth and $\frac{C-A}{C}=0.000629$. The uncertainty $(\cdot 03$ or $\cdot 04)$ of the results arises from Brown's transformation from rectangular to polar co-ordinates; the transformation is now being recomputed.

7. Hopmann gave an account of a study he has made on the measures of absolute heights on the Moon. He has examined the four existing catalogues for which Table I gives in the first four columns, the author, measurer, number of plates, and the number of heights measured, respectively.

Table I

\begin{tabular}{|c|c|c|c|c|c|c|c|}
\hline Author & Measurer & Pl. & Obj. & $\hbar$ & [vv] & m.s.e. & Stan. dev. \\
\hline Schrutka I & Franz & 5 & 150 & -0.3 & $31 \cdot 57$ & $\pm 1 \cdot 5$ & $\pm \quad I .9$ \\
\hline Schrutka II* & Haidrich & 8 & I35 & $-1 \cdot 4$ & $39 \cdot 64$ & $\mathrm{I} \cdot 8$ & I. 8 \\
\hline Baldwin & Baldwin & 5 & 696 & -0.5 & $3^{8} \cdot 34$ & $2 \cdot 1$ & $2 \cdot I$ \\
\hline U.S. Army $\mathrm{N}$ & ap Services & 15 & 256 & $+1 \cdot 5$ & 29.96 & $\mathrm{r} \cdot 9$ & $2 \cdot I$ \\
\hline
\end{tabular}

* Not yet published.

Of all the objects measured, only 18 are common to all four catalogues. $h$ in Table $\mathbf{I}$ is the mean height of these 18 points and $v=h-\hbar$. It would appear that the four series are of about the same quality. For these 18 objects the mean of the mean square error for a single series is $\pm \mathbf{I} \cdot 60 \mathrm{~km}$ or $\pm 0.80 \mathrm{~km}$ for the mean of the four series. However, the standard deviation of the heights is $\pm 0.86 \mathrm{~km}$, so that these $\mathrm{I} 8$ points, which are the best measures, give no significant indication of a deviation of the surface of the Moon from a sphere.

The heights of objects common to more than one catalogue were then compared statistically and the results are given in Table 2 , where $Z$ is the number of common objects and $r$ the correlation coefficient.

\section{Table 2}

Combination

Schrutka I - Schrutka II

Schrutka I - Baldwin

Schrutka I - A.M.S.

Schrutka II - Baldwin

Schrutka II - A.M.S.

Baldwin - A.M.S.

$\begin{array}{rc}Z & r \\ 54 & +0.069 \\ 68 & +0.163 \\ 59 & +0.453 \\ 69 & +0.172 \\ 60 & +0.003 \\ 106 & +0.072\end{array}$

m.s.e.

$\pm 0.120$

o. 118

0.103

0.118

0.129

0.097 
In these $4 \mathrm{I} 6$ comparisons, there were 35 cases of individual heights differing by $5 \mathrm{~km}$ or more and 12 by $6 \mathrm{~km}$ or more. For IAU Nr. $35 \mathrm{I} 6=$ Apianus a difference of $\mathrm{I} 8 \mathrm{~km}$ was found between Schrutka II $(-13.1 \pm 2.6 \mathrm{~km})$ and A.M.S. $(+4.9 \pm 0.9 \mathrm{~km})$. It would therefore appear that all the measured heights are unreliable, the heights being less than their uncertainties, although relative heights are quite good.

At present the available material is not good enough to derive a real deviation of the figure of the Moon from a sphere. It might be possible to form a General Catalogue from the four series by reducing them all to the same system, perhaps A.M.S. or Schrutka I. New and better material may be required as for example that already described by Arthur. Hopmann himself has been trying his new method of measuring points just appearing or disappearing together with the time and position of the terminator, with visual micrometers. From the 29 points so far measured, comparison with the second contour map of the Army Map Service gives a correlation coefficient of $+0.78 \pm 0.06$ and a mean difference of $\pm 0.89 \mathrm{~km}$.

C. L. Goudas gave a brief introductory account of the work published in Icarus, December I 963 , and went on to describe the extension of this work carried out since. The lunar surface has been considered as a series of spherical harmonics using the absolute heights of SchrutkaRechtenstamm together with the absolute heights of an additional $3^{2}$ points near the limb. The other side of the Moon has been considered as symmetrical. He showed the formulae used. It had previously been shown that the second harmonic did not stand out and therefore an ellipsoid is no better representation of the lunar surface than a sphere. It now appears that the fourth harmonic is greater than the second while the third is small and the fifth negligible.

In the discussion which followed, Hopmann again stressed that he considered the fundamental data subject to great uncertainty. O'Keefe asked about the homogeneity test and pointed out that Schrutka did not use the centre of gravity but a point about $\mathrm{I} \mathrm{km} \mathrm{distant.}$

K. Koziel described his determination of the libration constants from the four heliometric series covering the period $1877-1915$. The results obtained have already been published on page 217 of the Draft Reports 1964 . He stressed the reliability of his values for $I$ and $f$ and pointed out the exactitude of the proof for the uniqueness of the solution for $f$ as well as the simultaneous determination of the constants of forced libration together with those of free libration in longitude; moreover he stressed that an exact solution of the libration problem can only be obtained by using observations covering a long period.

In the discussion which followed, Kopal congratulated the President on having been able to solve the difficult problem of obtaining a reliable value for $f$.

$C$. F. Martin described the work on which he is currently engaged using lunar occultation observations made during the period $162 \mathrm{I}-\mathrm{I} 85^{\circ}$. There are $\mathrm{I} 178$ observations of which many have not been used before. They will be reduced using the Improved Lunar Ephemeris and if possible limb corrections from Watts's charts will be applied. The reduced observations will be used to investigate the rotation of the Earth and derive values of $\Delta T$. 